\title{
RNAi Vectors for Manipulation of Gene Expression in Higher Plants
}

\author{
Sayaka Hirai and Hiroaki Kodama*
}

\author{
Graduate School of Horticulture, Chiba University, 648 Matsudo, Chiba 271-8510, Japan
}

\begin{abstract}
RNA interference (RNAi) is a homology-dependent gene silencing technology in which small interfering RNAs (siRNAs) direct RNA cleavage or DNA methylation. After transcription of an RNAi cassette including inverted repeat sequences against the target gene and a spacer fragment, the resultant transcript forms a hairpin-like structure. The stem region of hairpin RNAs is processed into siRNAs. Here we focus on the structural properties of RNAi vectors that affect the silencing efficiency, and caveats in the evaluation of RNAi phenotype are discussed. Subsequently, several RNAi applications including simultaneous silencing of multiple gene sequences and specific silencing of a member in the gene family were discussed. In addition a newly developed RNAi technology, artificial microRNA, is also introduced.
\end{abstract}

\section{INTRODUCTION}

RNA interference (RNAi) discovered by Fire et al. [1] can ectopically reduce the expression of a target gene. RNAi-based techniques have been employed as effective experimental tools for the elucidation of gene function. According to the accumulation of genomic sequence information of various plants species, the analysis of functionally unidentified genes has been dramatically increasing. Such efforts enable identification of the genes conferring valuable traits. Inactivation of undesirable genes has also been reported in the breeding of plants with increased values for nutrition and food processibility. For example, RNAi has been applied to the production of cotton seeds with highstearic and high-oleic acids [2, 3], soybean producing seed oil with increased stability at high temperatures [4], tomato fruits with increased carotenoid and flavonoid content [5] and high lysine corn for animal food [6]. Since RNA silencing (including RNAi) share a common mechanism with plant defense mechanisms against invasive viruses, RNAi was used for developing plant immunity as soon as the RNAi vector system was available [7]. Flower quality is also the target of RNAi-based breeding. Rapeseeds with increased flowers [8] and roses with blue-coloured petals [9] were produced by using RNAi technology.

RNAi is a conserved mechanism in a wide range of eukaryotic organisms except for Saccharomyces cerevisiae [10, 11]. RNAi inactivates gene expression in a sequence-specific manner. Double-stranded RNA (dsRNA) is an effective trigger of RNAi. In mammalian cells, nematodes and flies, RNAi can be induced by direct introduction of dsRNAs. In contrast, RNAi in plants is usually established by transformation with a construct that produce hairpin RNAs. Chuang and Meyerowitz [12] first demonstrated that efficient RNA silencing in Arabidopsis thaliana can be conferred by an RNAi construct. They showed that introduction of an RNAi vector resulted in the silencing of a homeobox gene equivalent to that of the corresponding null mutant. The plant

\footnotetext{
*Address correspondence to this author at the Graduate School of Horticulture, Chiba University, 648 Matsudo, Chiba 271-8501, Japan;

E-mail:kodama@faculty.chiba-u.jp
}

RNAi vector consists of an inverted repeat harboring target sequences under the control of a strong promoter. The inverted repeat sequences are separated by a spacer fragment. After transcription, inverted repeat sequences form a dsRNA structure (the so-called stem) and a spacer forms the loop of a hairpin RNA, respectively (Fig. 1). RNase III enzymes called Dicer or Dicer-like protein (DCL) process dsRNAs into $21 \sim 25$-nt-long small interfering RNAs (siRNAs) [1315]. Arabidopsis has four DCL genes $(D C L 1 \sim D C L 4)$. In most RNAi plants, dsRNAs are produced under the control of a strong promoter, and resulting high dsRNA levels bypass the true genetic requirement of RNAi and activate different RNAi-related pathways. Thus, partially redundant DCL activities are apparently involved in the plant RNAi. Indeed, Dunoyer et al. $[16,17]$ showed that $D C L 4$ is specifically required for 21-nt siRNA production from the moderately expressed transgene. Then siRNAs are incorporated into RNA-induced silencing complex (RISC) which consists of several proteins including the Argonaute (AGO) protein. RISC is presumably located in the P-body, an RNA degradation center in the cytoplasm [18]. In Arabidopsis, ten predicted AGO family members $(A G O 1 \sim 10)$ have been identified. The enzyme responsible for mRNA cleavage in RNAi is called a slicer. AGO1 is the main slicer involved in RNAi, and its binding to siRNAs and mRNA cleavage activity were demonstrated in vitro [19]. After RISC-mediated mRNA cleavage, the resulting degraded products are further subjected to the exonucleolytic degradation [20,21].

Mette et al. [22] showed that transcription of inverted repeat sequences against the nopaline synthase promoter caused cytosine methylation of the corresponding promoter region in the genome. This RNA-directed DNA methylation (RdDM) is followed by the abolishment of transcriptional activity of the methylated promoter. Requirement of a histone deacetylase in RdDM suggests that RdDM is associated with heterochromatinization of the targeted promoter region $[23,24]$. In fact, siRNAs against the centromere region are essential for formation and maintenance of the heterochromatin state of the centromere [25]. The pattern of dimethylation at $\mathrm{H} 3 \mathrm{~K} 9$, a heterochromatic maker, also changed in the rice RdDM [26]. Condensation of chromatin blocks the interaction of trans-acting factors with a cis-acting element in 


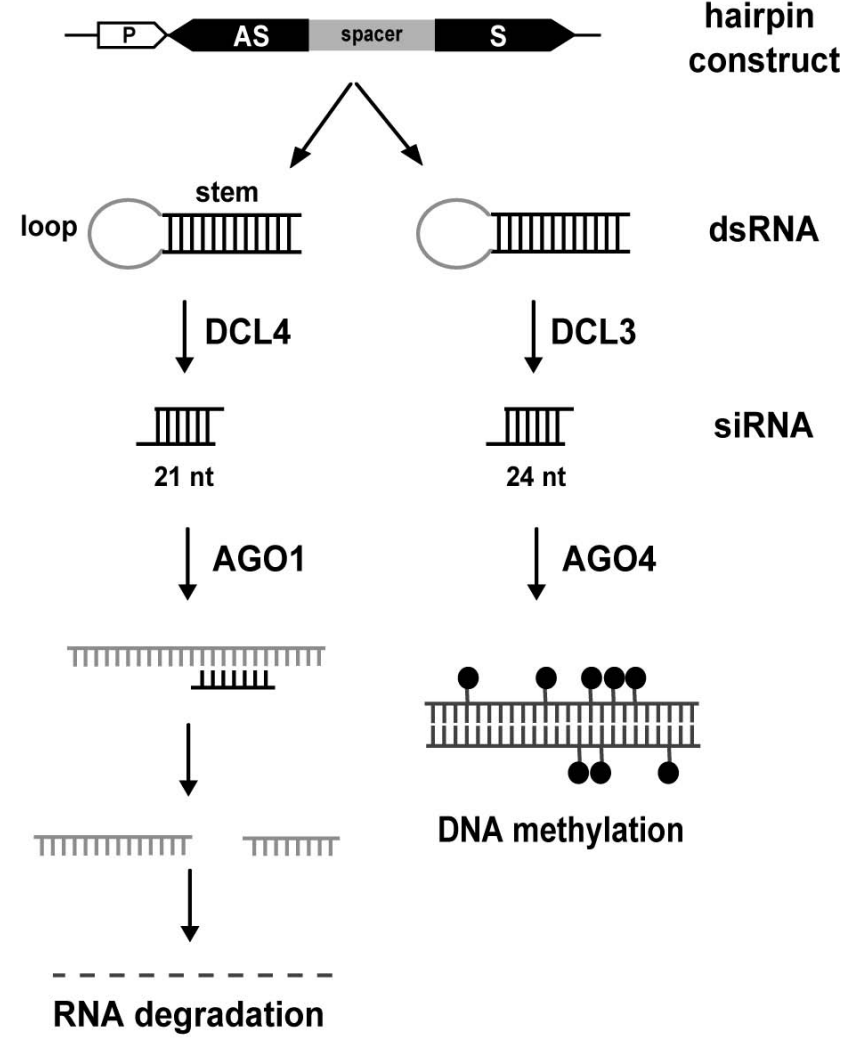

Fig. (1). Plant silencing pathways induced by an RNAi vector. An RNAi construct produces long dsRNAs after transcription. Two distinct DCL enzymes process this dsRNA. DCL3 processes dsRNAs into 24-nt-long siRNAs which direct cytosine methylation on the homologous genomic loci. DCL4 probably produces 21-ntlong siRNAs. The siRNA incorporated into AGO1 guides the endonucleolytic cleavage of homologous RNA, and siRNAs at AGO4 guide the methylation of cytosine in the complementary genomic sequences. P, promoter; AS, antisense oriented target sequence; $\mathrm{S}$, sense-oriented target sequence.

the promoter. More than 20 Arabidopsis genes, such as AGO4, DCL3, etc., are considered to be involved in RdDM [27]. The sequence specificity for cytosine methylation is likely determined by a specific class of 24-nt long siRNAs $[25,28]$.

Taken together, RNAi can potentially repress the expression of target genes by two different pathways; one is via mRNA cleavage and the other is via transcriptional inactivation. In plants, both silencing pathways can be controlled by the introduction of RNAi vectors. In this review, we address technical aspects of RNAi vector-mediated control of gene silencing in plants; namely the structural properties of RNAi vectors required for efficient silencing and several applications with RNAi vectors in plants. Readers should refer to several other reviews for the detailed mechanisms of RNAi $[15,29,30]$.

\section{DESIGNING RNAI VECTORS FOR EFFICIENT SILENCING}

\section{Choice of Target Region}

The inverted repeat region against the target gene forms a dsRNA structure which serves as the substrate of Dicer.
Thus the sequences and length of the stem region directly affect RNAi efficiency. Which part of a target gene should be chosen for acquisition of maximum silencing effect? Wesley et al. [31] constructed inverted repeat sequences against two different regions of a cDNA encoding an Arabidopsis flowering repression protein. One construct contained an entire cDNA sequence and another harbored the $3^{\prime}$ twothirds of the cDNA. Almost all transformants with these two RNAi cassettes flowered earlier than the wild-type plants. In contrast, Heilersig et al. [32] showed that the 3' sequences were a less efficient silencing inducer than the sequences from the $5^{\prime}$ and middle region of the rice starch synthase gene, GBSSI. There are few reports examining the relationship between the location of target region and resulting RNAi efficiency. At least to our knowledge, there are no genes harboring the region resistant to RNAi. As mentioned later, selection of the target region would be an important factor determining the specificity of RNAi.

RNAi efficiency has been investigated in terms of the length of the stem region. In animal cells, long dsRNAs induce the interferon response that eventually leads to cell death [33]. To avoid this cellular response, short hairpin RNA molecules with a 25 30-nt long stem have been used. Direct introduction of dsRNA into cells is effective for induction of RNAi in higher plants [34]. However, in most cases of plant RNAi, hairpin constructs that produce transcripts with a long stem region (about 100 1,000-nt in length) have been used. These RNAi vectors are premised on the insertion of cDNA fragments that are easy to handle (Table 1). The bottom and upper threshold of the cDNA length in the effective size range is about $100 \mathrm{nt}$ and $1,000 \mathrm{nt}$, respectively. Heilersig et al. [32] investigated RNAi efficiency in potato plants transformed with several RNAi vectors harboring inverted repeats with different sizes (488 1,300-nt in length). A stem region longer than 1,100 nt was less effective in silencing. The longest length of stem region showing successful silencing is $1,492 \mathrm{nt}$ of the $5^{\prime}$ chalcone synthase $(C H S)$ gene in petunias [35]. The most frequently chosen length of the stem region is about 200 500 nt, and these hairpin constructs showed effective silencing in various plant species including monocotyledonous ones [36].

\section{Choice of Spacer Sequence}

The spacer sequence allows us to construct an RNAi vector more easily, since replication of inverted repeat sequences without a spacer is unstable in Escherichia coli. Spacer sequences have been prepared from the $\beta$ glucuronidase $(G U S)$ gene [12], green fluorescence protein $(G F P)$ gene [37], and several introns [31, 38-41]. The enhanced RNAi effect can be often observed by using an intron spacer rather than the GUS or GFP spacers [31, 38, 42]. One possible reason for the enhancement of intron-mediated silencing is the splice-activation effect. The self-annealing of sense and antisense region of the transcript would be promoted after removing the intronic spacer sequences, and the resulting dsRNA molecules would be effectively transported into cytoplasm via nucleocytoplasmic transport receptors [43]. Smith et al. [38] demonstrated that almost $100 \%$ of plants transformed with an intron-containing hairpin construct showed silencing. This report had a very strong impression on many researchers, following which RNAi cassettes were frequently constructed by using an intron spacer. 
Table 1. Stem Length of the RNAi Vector and Silencing Efficiency

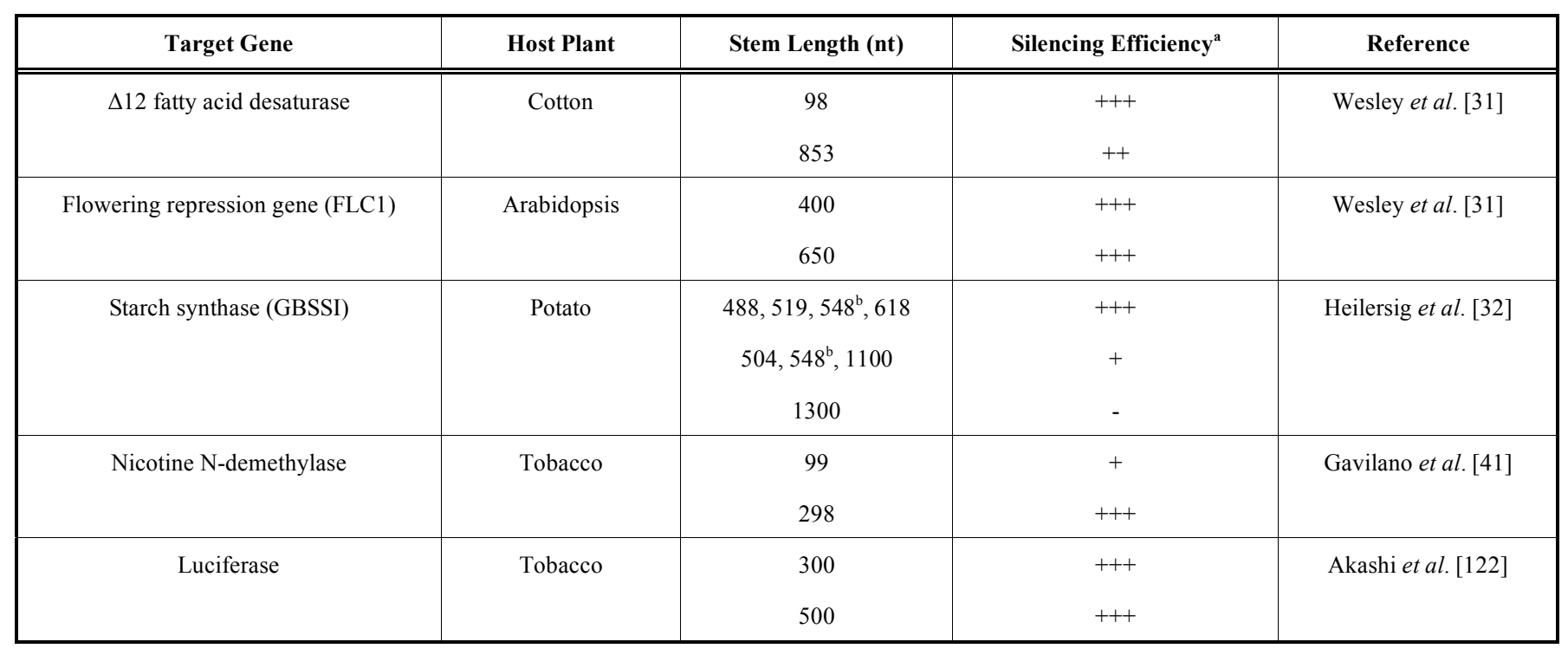

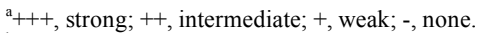

these two stems harbor the sequences with same length but they correspond to distinct region of the target gene.

Several intronic sequences, not all, have been shown to enhance the expression of the cognate genes as a cis-acting element [44-47]. The ubiquitin promoter in combination with the intron gave a high expression of downstream genes in rice [48-50]. In fact, an intron of the maize ubiquitin gene was placed $5^{\prime}$ upstream of inverted repeat sequences in order to enhance dsRNA expression in rice [51]. Thus, it is also possible that the intron spacer strengthens the RNAi effects by intron-mediated enhancement in the cognate transcript level and not by the splice-dependent promotion of selfannealing. Hirai et al. [52] investigated the effect of intron spacer on RNAi efficiency using a hairy root assay system. The 1st intron of the NtFAD3 gene encoding a tobacco $\alpha$ linolenate synthase gave a moderate silencing efficiency, even though the splicing itself was efficient [52]. Similar or less silencing efficiency of RNAi cassettes using an intron spacer has been reported in comparison with cassettes using non-intronic sequences [39, 53, 54]. These results indicate that the use of intronic sequences as a spacer is not always required for the establishment of efficient silencing.

The relationship between spacer length and silencing efficiency was also investigated by using a hairy root assay system [52]. Several GUS spacers with different lengths ranging from 100 to $1800 \mathrm{nt}$ were used in the construction of RNAi vectors. Although these RNAi constructs have the same stem region, they conferred different silencing efficiencies. The strongest RNAi effect was obtained in RNAi constructs with 500- and 1,000-nt GUS spacers and not a 100-nt one [52]. The RNAi construct against the tomato ACC oxidase gene with a short spacer ( $7 \mathrm{nt}$ in length) conferred an efficient silencing than that with a 1,002-nt spacer sequence [55]. In contrast, 1,100-bp and 150-bp-long spacers showed nearly the same silencing efficiency of a GBSSI gene in potato plants [32]. In conclusion, the spacer sequence certainly influences the silencing efficiency, but we cannot predict which RNAi cassettes can confer strong or weak silencing when different spacers are used. When an RNAi cassette against the NtFAD3 gene was constructed using a 100-nt
GUS spacer, the resulting moderate silencing was associated with the accumulation of its hairpin RNA. Thus moderate silencing was attributed to an inefficient processing of its hairpin RNA by Dicer [52]. In most cases, hairpin RNA is rapidly degraded into siRNA and its steady-state level is very low. If different silencing degrees are required, use of several spacers with different lengths or different sequences are one of the meaningful approaches of RNAi.

\section{Promoter Sequences and Other cis-Acting Elements}

The degree of silencing is apparently proportional to the level of siRNAs, and "strong" promoters have been used in the construction of RNAi vectors as follows, the cauliflower mosaic virus $35 \mathrm{~S}$ promoter (p35S) [41, 56, 57], soybean lectin promoter [58], Arabidopsis rbcS promoter [59, 60], rice ubiquitin promoter $[61,62]$ and Chrysanthemum rbcS1 [63]. In addition, the cis-acting elements that potentially assist in the transcription of an RNAi cassette have been used. One such element is the intronic sequence (see also previous section) [51]. Another possible cis-element is the matrix attachment region (MAR). As was the case of the sense transgene, expression of the RNAi construct should suffer position effects. Transgenes integrated into or near the highly condensed chromatin regions often undergo condensation, resulting in a reduction of transcription. MARs are AT-rich sequences that bind specifically to the nuclear matrix and potentially reduce or eliminate position effects [64-67]. Insertion of a MAR identified from the human interferon $\beta$ gene into the short hairpin construct enhanced the stability and degree of RNAi in animal cells [68]. Li et al. [60] investigated the effect of a MAR derived from the tobacco $r b 7$ gene on RNAi efficiency and stability. However, this MAR had no discernible influence on silencing in transgenic poplar plants. Since MARs with no effects on the reduction of variability in transgene expression levels have also been reported [69-72], there is room to improve RNAi efficiency if the MARs from different sources are incorporated into RNAi vectors. 


\section{siRNA Sequences Generated from the Hairpin RNA}

Llave et al. [73] determined the siRNA sequences that had been generated from the transiently expressed RNAi cassette against the GFP gene. The distribution of cloned siRNA sequences showed that some siRNAs partially shared their sequences with other different siRNA clones. Some clustering of siRNAs along the stem region was observed, indicating that siRNAs are apparently non-randomly distributed. One most likely explanation for this asymmetry in siRNA production is that each arm of a nascent hairpin RNA carries the folded regions. These highly structured regions are recognized as a small hairpin structure, and then will be processed by the plant DCL. Similar asymmetry in siRNA generation has been observed in the plant virus-induced RNA silencing [74]. Another possible explanation is that loading of small RNAs into AGO proteins is specified by the 5' terminal nucleotide. In fact, most 21-nt small RNAs with 5 ' terminal $\mathrm{U}$ were predominantly associated with AGO1 [75]. The asymmetry in the siRNA population may result from poor incorporation of siRNAs with 5' terminal nucleotide other than $U$, enhancing their turnover.

\section{Assay System for Evaluation of RNAi Efficiency}

When RNAi is used in the functional analysis of indispensable genes for life, severe silencing causes a lethal phenotype. Control of the degree of silencing may be required in such a case, and an assay system for the comparison of silencing efficiency among the RNAi vectors would be valuable for this purpose. Hellens et al. [76] developed a transient assay for quantification of RNA silencing. In this assay, an RNAi vector is introduced into tobacco leaf cells by agroinfiltration. The inverted repeat sequences against the firefly luciferase (LUC) gene and two independent expression constructs of $L U C$ and Renilla luciferase (REN) genes are included in a single T-DNA. The degree of silencing can be assessed in a few days by measuring the decrease of chemiluminescence of LUC relative to that of REN. This system would be valuable for the analysis of RNAi vector structures themselves, but was not suitable for elucidation of the degree of silencing of specific target gene sequences. Panstruga et al. [40] developed an assay that is based on transient expression using two fluorescent proteins as a marker (GFP) for transfected cells and the second (red fluorescent protein; RFP) as translational fusion with the target gene. Co-introduction of this test plasmid with an RNAi vector against the target gene resulted in a specific decrease of RFP fluorescence. This assay allows us to evaluate the degree of silencing of the target gene, and would be valuable for the design of RNAi vectors that confer a severe and mild trigger of RNAi. These methods can be used as an easy and rapid tool to analyze the effectiveness of RNAi without longterm plant-transformation experiment. If the phenotype of a target gene can be determined using root cells, the assay system using hairy roots provides the strict evaluation of the effects of RNAi constructs on target gene expression in a relatively short experimental term $[52,77]$.

\section{GENERATION OF IMPROVED RNAI VECTORS}

\section{High-Throughput RNAi Vectors}

Construction of RNAi vectors is a time-consuming and laborious work since two fragments corresponding to the antisense and sense region of the stem should be subcloned into a binary vector. To inactivate multiple gene functions, a rapid and easy method for construction of RNAi vectors is certainly helpful. High-throughput plant RNAi vectors have been constructed to fulfill such a demand. For instance, single step construction of an RNAi vector has been developed. pHELLSGATE [78] and pANDA [51] vectors allow the assembly of an inverted repeat structure by Gateway recombination technology [79-81]. In the construction of these RNAi vectors, PCR products for the target gene are generated with primers flanking attB1 and attB2 sites, and then recombined into two cloning sites with attP1 and attP2 sequences by BP clonase. Although this single step reaction was easy to construct, the resulting long recombination-related sequences weakened the silencing efficiency [82]. Therefore, a two-step recombination procedure, which leaves short sequences on the resulting RNAi vector, has been recommended for efficient silencing [81]. Several pHELL SGATE-related RNAi vectors have been developed for RNAi in monocotyledonous plants and for inducible RNAi [79].

For conventional cloning, pHANNIBAL [31], pKANNIBAL [83], pSAT [84] and pSH [52] are available. In these RNAi vectors, PCR fragments of the target gene are produced by using primers with restriction sites, and cloned successively into both upstream and downstream region of the spacer to become the two arms of the hairpin construct.

\section{Inducible RNAi Vectors}

RNAi technique cannot be applied to genes whose silencing interferes with plant regeneration or causes embryo lethality or severe pleiotropic phenotypes. For example, RNAi against the Arabidopsis Zinc finger transcription factor gene, ZAT6, was lethal [85]. Complete depletion of $\gamma$-tubulin by RNAi was lethal at the cotyledon stage [86]. To counter this problem, the lethal and sterile phenotypes are eluded by using an inducible RNAi vector which can confer transient and local silencing. Ethanol or estrogens are used as chemical regulators of these inducible promoters. In the case of an ethanol-inducible vector, a transcriptional regulator, AlcR, is constitutively expressed and the RNAi cassette is inserted behind the alcA promoter. After ethanol treatment, AlcR binds to the alcA promoter and transcription of the downstream RNAi sequences is activated [87].

Another inducible RNAi vector is developed by the combination of Cre/loxP-mediated recombination and a chemical inducing system, in which the stringent control of expression of an RNAi cassette can be achieved. A chimeric transcriptional factor, XVE, is constitutively expressed. When XVE binds to estrogens, the activated XVE induces the transcription of a Cre recombinase gene. The resulting Cre recombinase removes a fragment that blocks transcription of an RNAi cassette [88].

The pHELLSGATE vector-based inducible RNAi vector is also available $[89,90]$. This system uses a modified RNAi cassette from pHELLSGATE under the control of the pOp6 promoter. A synthetic transcription factor, LhGR, is constitutively expressed by $\mathrm{p} 35 \mathrm{~S}$. In the presense of dexamethasone, LhGR binds to pOp6 and the downstream RNAi cassette is transcribed. These inducible RNAi vector systems allow the rapid induction of RNAi across the whole plant or in limited tissues under investigation [91]. 


\section{amiRNA}

A new RNAi vector was recently developed in which the structure of hairpin RNA mimics that of microRNAs (miRNAs). This new RNAi vector is called as artificial microRNAs (amiRNAs) [92]. The precursor RNA of miRNAs is transcribed from the genomic bona fide loci distinct from the locus encoding target RNA [93, 94]. miRNA precursors show a characteristic feature: mismatches or bulges are included in the stem. One miRNA-miRNA* duplex (* means a dispensable RNA strand in miRNA-mediated silencing) is produced after processing by DCL. In the amiRNA vector, the target sequence was inserted as miRNA sequences and the complementary sequence was designed as miRNA* $[92$, 95]. The position and number of mismatches and bulges in amiRNA closely resemble a representative miRNA. The single small RNA produced from an amiRNA has a few mismatches against the target RNA. The $3^{\prime}$ terminal base of resulting small RNAs is not homologous to target mRNA by avoiding the nucleotide extension by an RNA-dependent RNA polymerase [92].

For designing amiRNAs, WMD (Web MicroRNA Designer) platform based on a set of provided criteria is available [96]. Qu et al. [97] demonstrated that an amiRNA against the $2 \mathrm{~b}$ gene of Cucumber mosaic virus (CMV) efficiently conferred resistance to $\mathrm{CMV}$ infection on a tobacco plant. Since the amiRNA vector produces single small RNA species as a guide of RISC, the selection of a sequence corresponding to the miRNA region is extremely important as was the case of the construction of short hairpin RNAs in animal cells. If potential targets are properly predicted in the genome-wide range, non-specific silencing by amiRNAs is markedly reduced in comparison with the silencing via orthodox RNAi vectors. In addition, high specificity by amiRNAs can be adapted for allelic-specific inactivation. In this respect, amiRNA technology may be suitable for model plants such as Arabidopsis and rice. The Arabidopsis amiRNA library provided by Drs. Hanon, Martienssen, McCombie and their colleagues is now released on the market, in which each 22,000 gene is targeted by 3 different amiRNAs. These amiRNAs resemble the miR319a structure and they are transcribed under the control of p35S. The amiRNA vector is constructed by using a binary vector, and is ready to transform plants [98]. Arabidopsis amiRNAs would open up an easy way of specific inactivation of target genes.

\section{FUNCTIONAL KNOCKDOWN OF SINGLE GENE OR MULTIPLE GENE SEQUENCES}

Since sequence specificity is determined by hybridization of siRNAs to corresponding target mRNAs, siRNAs consisting of sequences shared by several genes cause simultaneous silencing of multiple gene sequences (Fig. 2a). Efficient silencing of several members of a gene family is demonstrated in the families for rice OsRac genes [61], Solanum americanum PIN2 [57], and the soybean $\omega-3$ fatty acid desaturase [4]. A single RNAi construct against the conserved region in topspoviruses can interfere with the infection of four different topspoviruses in tomato [99]. The nucleotide similarity that allows simultaneous silencing is important to estimate the effects of RNAi against the gene family member. In this respect, $\mathrm{Xu}$ et al. [100] showed that simultaneous silencing would occur between genes showing sequence identity with at least 22-nt continuous stretches.

In contrast, in order to analyze the function of specific member of a gene family without breaking down the expression of others, use of the $3^{\prime}$ untranslated region (UTR) is effective (Fig. 2b). Specific silencing against single member of tobacco psbP [101], torenia CHS [102], the Arabidopsis $P R F$ [103] and rice OsRac [61] gene families was achieved by using $3^{\prime}$ UTR sequences as a trigger of silencing. In addition, specific inactivation of a target gene can be carried out by using the amiRNA vector in Arabidopsis (Fig. 2c, see previous section).

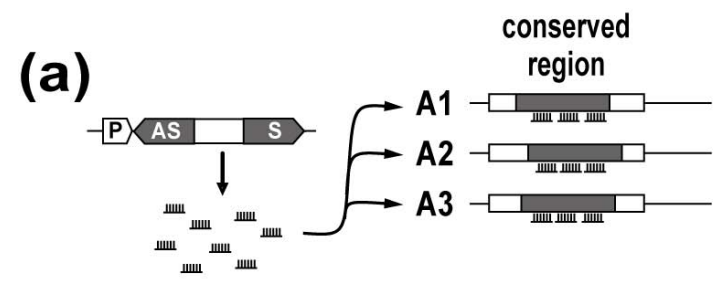

(b)

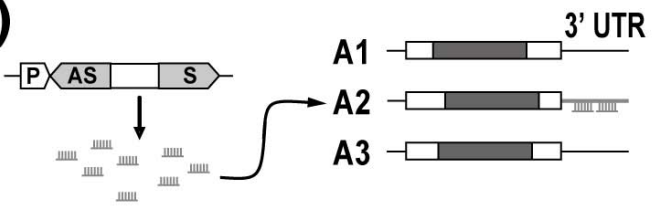

(c)

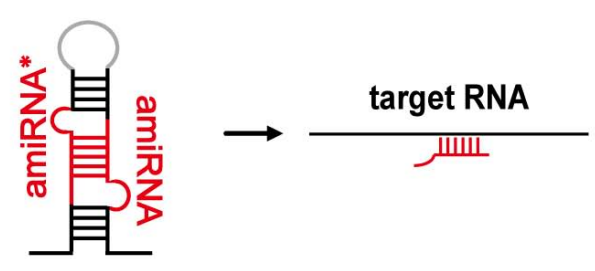

(d)

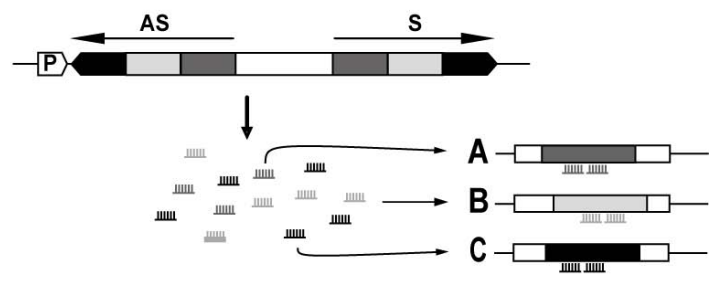

Fig. (2). mRNA Targeting by different strategies.

(a) When the conserved regions among the related gene sequences (referred to as A1 A3) is used for construction of an RNAi vector, siRNAs generated from the resultant RNAi vector can simultaneously inactivate the multiple target genes. (b) When the $3^{\prime}$ untranslated region (UTR) is used to generate an inverted repeat sequence, this RNAi vector can suppress the expression of a specific member of related genes. (c) The amiRNA vector produces a transcript that mimics the miRNA precursor. The miRNA and miRNA* are replaced with target gene sequences and corresponding complementary sequences, respectively. In the amiRNA vector, single small RNA guide the RISC. (d) To inactivate multiple target genes (each of them have unrelated sequences from the others; referred to as A, $\mathrm{B}$ and $\mathrm{C}$ ), a chimeric arm of the stem consisting of the target genes can be used. After processing, the siRNAs corresponding to the targeted genes are simultaneously produced at once. 
If the expression of two or more genes were to be suppressed, successive transformation would be required. However, there are only a limited number of selectable marker genes that are available for practical use. For simultaneous RNAi against several unrelated targets, Zhong et al. [104] constructed an RNAi vector in which two RNAi expression cassettes are tandemly arranged in single T-DNA. Similarly, conjugation of several RNAi cassettes onto a single binary plasmid was reported [84]. When these multiple RNAi cassettes were driven by the same promoter, the resulting transformants potentially have the risk of attenuating the RNAi due to the cross silencing in the same promoters. These problems may be overcome by incorporation of multiple target sequences into single RNAi cassette. Miki et al. [61] developed an RNAi construct harboring multiple trigger sequences in a single inverted repeat structure. They showed simultaneous successful silencing of double or triple target genes by single transformation (Fig. 2d). This method can save the promoter and selectable marker gene sequences.

\section{RNAi VECTOR FOR INDUCTION OF RDDM}

The structural properties of promoter hairpin RNA expression vectors have not been investigated well. p35S is silenced by promoter-targeted RNAi in petunia [35] and rice [26]. In petunia plants, promoter hairpin cassettes carrying a whole region of $\mathrm{p} 35 \mathrm{~S}$ and a 261-bp enhancer region showed efficient reduction of transcriptional activity, but an RNAi construct against the 123-bp minimal promoter for $\mathrm{p} 35 \mathrm{~S}$ showed limited repression of transcriptional activity [35]. We investigated the region conferring an efficient transcriptional silencing of $\mathrm{p} 35 \mathrm{~S}$ in tobacco plants. These constructs contained inverted repeats corresponding to four distinct regions of p35S (Fig. 3). Leaves of the transgenic tobacco which express the GUS gene under the control of $\mathrm{p} 35 \mathrm{~S}$ were re-transformed with Ri plasmid and these RNAi constructs. These four distinct RNAi vector reduced the GUS activity to similar levels, indicating that transcriptional activity of $\mathrm{p} 35 \mathrm{~S}$ can be repressed by using RNAi cassettes harboring about 200-bp-long promoter sequences (Fig. 3).

It is important for most of researchers to assess whether or not the siRNA can inactivate the promoters of endogenous genes. In this respect, only sporadic evidence has shown its validity. Inactivation of endogenous promoters by $\mathrm{RdDM}$ has been shown in petunia [35], Arabidopsis [105], maize [106], and potato [107]. In contrast, six of seven endogenous rice genes were resistant to RdDM-mediated promoter inactivation although de novo methylation itself occurred in these promoter sequences [26]. Since RdDM-associated transcriptional silencing is apparently influenced by the local surrounding genomic condition of the target promoter locus [108], reduction of promoter activity may significantly vary among the endogenous promoters of interest, ranging from no reduction to an almost complete knockdown level by the effects of the surrounding genomic sequences.

\section{OFF-TARGET EFFECT}

Recent investigations have demonstrated that transcripts having less than $100 \%$ complementarity with an siRNA can be cleaved by the RNAi pathway [109-111]. The downregulation of mRNAs other than the intended target in RNAi is referred to as off-targeting, which deteriorates the specificity and applicability of RNAi [112]. In fact, a computational (a)

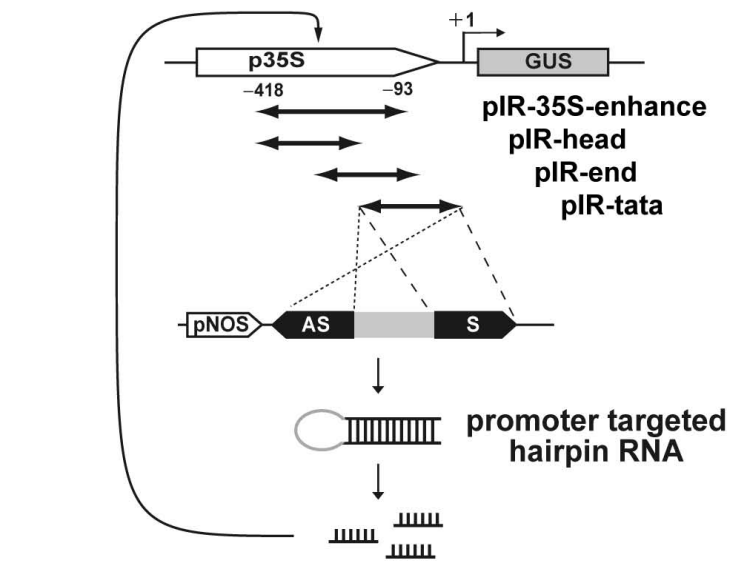

(b)

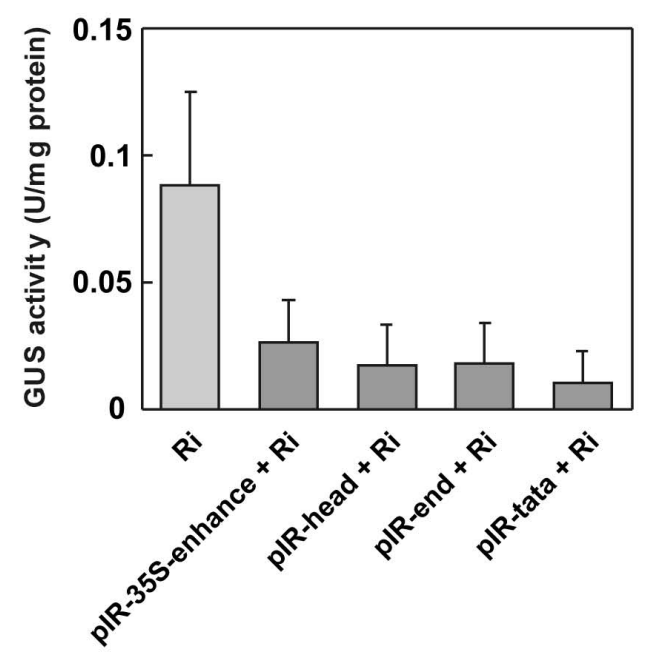

Fig. (3). RdDM-mediated inactivation of p35S.

The RNAi constructs against four different regions of $\mathrm{p} 35 \mathrm{~S}$ (a) and their RNAi phenotype (b). The inverted repeat sequence was transcribed by a nopaline synthase promoter (pNOS). The RNAi cassette is inserted into pSMAH621 (personally distributed by Dr. Ichikawa). Each RNAi vector contains an inverted repeat corresponding to the enhancer region $(-418 \sim-93$ from the transcriptional start point, pIR-35S-enhance). $5^{\prime}$ region (-418 -219, pIRhead), central region (-293 -94, pIR-end), or 3' most region containing the TATA box $(-200 \sim+1$, pIR-tata). These RNAi vectors and an Ri plasmid were introduced into transgenic tobacco leaves expressing the GUS gene under the control of $\mathrm{p} 35 \mathrm{~S}$ (a representative line, G36). The hairy roots were induced on Murashige-Skoog medium supplemented with 3\% sucrose, $0.3 \%$ Gellan gum, 750 $\mathrm{mg} / \mathrm{L}$ Augmentin (GlaxoSmithKline) and $10 \mathrm{mg} / \mathrm{L}$ hygromycin B. The GUS activity of each hairy root was determined using an AURORA GUS kit (ICN Biomedicals) with a LUMAT LB9506 luminometer (Berthold) by counting relative chemiluminescence units. The GUS enzyme unit was calculated by using Ampullaria $\beta$ glucuronidase (Wako Pure Chemical Industries) as a standard. Vertical lines indicate SD $(n=10)$. These RNAi constructs reduced the GUS activity by about $68 \% \sim 88 \%$ when compared with those of control hairy roots.

study using the genome and transcriptome sequence data of human, worm and yeast suggested that the risk of off-target 
effects by siRNAs cannot be ignored $[111,113]$. The Jackson group found a way out of this problem to reduce unintended down-regulation in animal cells, which includes chemical modification in siRNAs that prevented the incorporation of sense siRNA into RISC, reducing siRNA concentration, and applying more rigorous bioinformatics to siRNA design $[114,115]$. As mentioned in a previous section, the off-target effect by amiRNA is potentially reduced when compared with that of a traditional RNAi vector. In contrast, preparation of experimental evidence of the off-target silencing by a traditional RNAi vector is quite difficult because of the complexity of siRNA species generated from hairpin RNAs with a long stem. In fact, different phenotypes, which may be due to the off-target effect, are observed between the null mutant and RNAi transformants [116]. To confirm that an RNAi phenotype is due to the "on-target" silencing, Thomas et al. [117] investigated the phenotypic variation among plants that had been transformed with two or more RNAi vectors against different regions of a single target mRNA. Kumar et al. [118] investigated whether or not the RNAi phenotype can be complemented by a synthetic gene that encodes the same protein as the native gene but uses different nucleotide sequences. If the phenotype of interest is due to on-target silencing, it will be completely reversed by expression of the synthetic gene. For RNAi to become a more reliable technology in plants, specificity and functionality of RNAi must be properly addressed.

Another potential factor of the off-target effect is the spreading of RNAi target by the amplification of siRNAs. This amplification process (termed transitivity) results from the action of RNA-dependent RNA polymerase [119]. Although transitivity may increase the incidence of off-target inactivation, it rarely occurs against endogenous genes [61, $120,121]$. This characteristic feature alleviates the off-target effects in RNAi analyses against endogenous genes.

\section{CONCLUSION}

The development of RNAi technology confers an efficient knockdown of gene expression and it has replaced antisense technology or cosuppression that show less efficient silencing, namely low silencing frequency or weak depletion of target mRNA. Although it seems paradoxical, the RNAi vector systems that allow modulating the degree of silencing, namely weak or strong silencing, would be helpful for further fine investigation of gene function. In addition, an easy and simple method for validation of on-target silencing has been desired. Although there are a lot of subjects to be solved for popularization in wide-range plant species, the development of an amiRNA vector would provide a more sophisticated RNAi system. The rapid progress of RNAi technology may enable to control the expression of a number of genes at a once, and to regulate target mRNA at a level that corresponds to researchers' demands in the near future.

$$
\begin{array}{ll}
\text { ABBREVIATIONS } \\
\text { AGO } & =\text { Argonaute } \\
\text { amiRNA } & =\text { artificial microRNA } \\
\text { CHS } & =\text { chalcone synthase } \\
\text { CMV } & =\text { Cucumber mosaic virus } \\
\text { DCL } & =\text { Dicer like }
\end{array}
$$

$$
\begin{array}{ll}
\text { dsRNA } & =\text { double-stranded RNA } \\
\text { GFP } & =\text { green fluorescence protein } \\
\text { GUS } & =\beta \text {-glucuronidase } \\
\text { LUC } & =\text { firefly luciferase } \\
\text { MAR } & =\text { matrix attachment region } \\
\text { p35S } & =\text { Cauliflower mosaic virus 35S promoter } \\
\text { RdDM } & =\text { RNA-directed DNA methylation } \\
\text { REN } & =\text { Renilla luciferase } \\
\text { RFP } & =\text { red fluorescence protein } \\
\text { RISC } & =\text { RNA-induced silencing complex } \\
\text { RNAi } & =\text { RNA interference } \\
\text { siRNA } & =\text { small interfering RNA } \\
\text { UTR } & =\text { untranslated region } \\
\text { WMD } & =\text { Web microRNA designer }
\end{array}
$$

\section{REFERENCES}

[1] Fire A, Xu S, Montgomery MK, Kostas SA, Driver SE, Mello CC. Potent and specific genetic interference by double-stranded RNA in Caenorhabditis elegans. Nature 1998; 391: 806-11.

[2] Liu Q, Singh SP, Green AG. High-stearic and high-oleic cottonseed oils produced by hairpin RNA-mediated post-transcriptional gene silencing. Plant Physiol 2002; 129: 1732-43.

[3] Tang G, Galili G. Using RNAi to improve plant nutritional value: from mechanism to application. Trends Biotechnol 2004; 22: 4639.

[4] Flores T, Karpova O, Su X, et al. Silencing of GmFAD3 gene by siRNA leads to low $\alpha$-linolenic acids (18:3) of fad3-mutant phenotype in soybean [Glycine max (Merr.)]. Transgenic Res 2008; [Epub ahead of print].

[5] Davuluri GR, van Tuinen A, Fraser PD, et al. Fruit-specific RNAimediated suppression of DETI enhances carotenoid and flavonoid content in tomatoes. Nat Biotechnol 2005; 23: 825-6.

[6] Houmard NM, Mainville JL, Bonin CP, Huang S, Luethy MH, Malvar TM. High-lysine corn generated by endosperm-specific suppression of lysine catabolism using RNAi. Plant Biotechnol J 2007; 5: 605-14.

[7] Wang H, Hao J, Chen X, et al. Overexpression of rice WRKY89 enhances ultraviolet $\mathrm{B}$ tolerance and disease resistance in rice plants. Plant Mol Biol 2007; 65: 799-815.

[8] Byzova M, Verduyn C, De Brouwer D, De Block M. Transforming petals into sepaloid organs in Arabidopsis and oilseed rape: implementation of the hairpin RNA-mediated gene silencing technology in an organ-specific manner. Planta 2004; 18: 379-87.

[9] Katsumoto Y, Fukuchi-Mizutani M, Fukui Y, et al. Engineering of the rose flavonoid biosynthetic pathway successfully generated blue-hued flowers accumulating delphinidin. Plant Cell Physiol 2007; 48: 1589-600.

[10] Grewal SIS, Moazed D. Heterochromatin and epigenetic control of gene expression. Science 2003; 301: 798-802.

[11] Kato A, Endo M, Kato H, Saito T. The antisense promoter of AtRE1, a retrotransposon in Arabidopsis thaliana, is activated in pollens and calluses. Plant Sci 2005; 168: 981-6.

[12] Chuang C-F, Meyerowitz EM. Specific and heritable genetic interference by double-stranded RNA in Arabidopsis thaliana. Proc Natl Acad Sci USA 2000; 97: 4985-90.

[13] Baulcombe D. RNA silencing in plants. Nature 2004; 431: 356-63.

[14] Matzke MA, Birchler JA. RNAi-mediated pathways in the nucleus. Nat Rev Genet 2005; 6: 24-35.

[15] Brodersen P, Voinnet O. The diversity of RNA silencing pathways in plants. Trends Genet 2006; 22: 268-80.

[16] Dunoyer P, Himber C, Voinnet O. DICER-LIKE4 is required for RNA interference and produces the 21-nucleotide small interfering RNA component of the plant cell-to-cell silencing signal. Nat Genet 2005; 37: 1356-60.

[17] Dunoyer P, Himber C, Ruiz-Ferrer V, Alioua A, Voinnet O. Intraand intercellular RNA interference in Arabidopsis thaliana requires 
components of the microRNA and heterochromatic silencing pathways. Nat Genet 2007; 39: 848-56.

[18] Parker R, Sheth U. P bodies and the control of mRNA translation and degradation. Mol Cell 2007; 25: 635-46.

[19] Baumberger N, Baulcombe DC. Arabidopsis ARGONAUTE1 is an RNA slicer that selectively recruits microRNAs and short interfering RNAs. Proc Natl Acad Sci USA 2005; 102: 11928-33.

[20] Valencia-Sanchez MA, Liu J, Hannon GJ, Parker R. Control of translation and mRNA degradation by miRNAs and siRNAs. Gene Dev 2006; 20: 515-24

[21] Belostotsky D. mRNA turnover meets RNA interference. Mol Cell 2004; 16: 498-500.

[22] Mette MF, Aufsatz W, van der Winden J, Matzke MA, Matzke AJ. Transcriptional silencing and promoter methylation triggered by double-stranded RNA. EMBO J 2000; 19: 5194-201.

[23] Aufsatz W, Mette MF, van der Winden J, Matzke AJ, Matzke M. RNA-directed DNA methylation in Arabidopsis. Proc Natl Acad Sci USA 2002; 4: 16499-506.

[24] Aufsatz W, Stoiber T, Rakic B, Naumann K. Arabidopsis histone deacetylase 6: a green link to RNA silencing. Oncogene 2007; 26: 5477-88.

[25] Onodera Y, Haag JR, Ream T, Nunes PC, Pontes O, Pikaard CS. Plant nuclear RNA polymerase IV mediates siRNA and DNA methylation-dependent heterochromatin formation. Cell 2005; 120: 613-22.

[26] Okano Y, Miki D, Shimamoto K. Small interfering RNA (siRNA) targeting of endogenous promoters induces DNA methylation, but not necessarily gene silencing, in rice. Plant J 2008; 53: 65-77.

[27] Matzke M, Kanno T, Huettel B, Daxinger L, Matzke AJ. Targets of RNA-directed DNA methylation. Curr Opin Plant Biol 2007; 10: 512-9.

[28] Qi Y, He X, Wang X, Kohany O, Jurka J, Hannon GJ. Distinct catalytic and non-catalytic roles of ARGONAUTE4 in RNAdirected DNA methylation. Nature 2006; 443: 1008-12.

[29] Vazquez-Ortiz G, Pina-Sanchez P, Salcedo M. Great potential of small RNAs: RNA interference and microRNA. Rev Invest Clin 2006; 58: 335-49.

[30] Ossowski S, Schwab R, Weigel D. Gene silencing in plants using artificial microRNAs and other small RNAs. Plant J 2008; 53: 67490.

[31] Wesley SV, Helliwell CA, Smith NA, et al. Construct design for efficient, effective and high-thoughput gene silencing in plants. Plant J 2001; 27: 581-90.

[32] Heilersig HJ, Loonen A, Bergervoet M, Wolters AM, Visser RG. Post-transcriptional gene silencing of GBSSI in potato: effects of size and sequence of the inverted repeats. Plant Mol Biol 2006; 60: 647-62.

[33] Watanabe T, Takeda A, Tsukiyama $\mathrm{T}$, et al. Identification and characterization of two novel classes of small RNAs in the mouse germline: retrotransposon-derived siRNAs in oocytes and germline small RNAs in testes. Genes Dev 2006; 20: 1732-43.

[34] An C, Sawada A, Fukusaki E, Kobayashi A. A transient RNA interference assay system using Arabidopsis protoplasts. Biosci Biotechnol Biochem 2003; 67: 2674-7.

[35] Sijen T, Vijn I, Rebocho A, et al. Transcriptional and posttranscriptional gene silencing are mechanistically related. Curr Biol 2001; 11: 436-40.

[36] Fu D, Uauy C, Blechl A, Dubcovsky J. RNA interference for wheat functional gene analysis. Transgenic Res 2007; 16: 689-701.

[37] Piccin A, Salameh A, Benna C, et al. Efficient and heritable functional knock-out of an adult phenotype in Drosophila using a GA14-driven hairpin RNA incorporating a heterologous spacer. Nucleic Acids Res 2001; 29: E55.

[38] Smith NA, Singh SP, Wang MB, Stoutjesdijk PA, Green AG, Waterhouse PM. Total silencing by intron-spliced hairpin RNAs. Nature 2000; 407: 319-20.

[39] Stoutjesdijk PA, Singh SP, Liu Q, Hurlstone CJ, Waterhouse PA, Green AG. hpRNA-mediated targeting of the Arabidopsis FAD2 gene gives highly efficient and stable silencing. Plant Physiol 2002; 129: 1723-31.

[40] Panstruga R, Kim MC, Cho MJ, Schulze-Lefert P. Testing the efficiency of dsRNAi constructs in vivo: A transient expression assay based on two fluorescent proteins. Mol Biol Rep 2003; 30: 13540.
[41] Gavilano LB, Coleman NP, Burnley LE, et al. Genetic engineering of Nicotiana tabacum for reduced nornicotine content. J Agric Food Chem 2006; 54: 9071-8.

[42] Lee YS, Carthew RW. Making a better RNAi vector for Drosophila: use of intron spacers. Methods 2003; 30: 322-9.

[43] Murchison EP, Hannon GJ. miRNAs on the move: miRNA biogenesis and the RNAi machinery. Curr Opin Cell Biol 2004; 16 223-9.

[44] Rose AB, Beliakoff JA. Intron-mediated enhancement of gene expression independent of unique intron sequence and splicing. Plant Physiol 2000; 122: 535-42.

[45] Joen JS, Lee S, Jung KH, et al. Production of transgenic plants showing reduced heading date and plant height by ectopic expression of rice MADS-box genes. Mol Breed 2000; 6: 581-92.

[46] Bourdon V, Harvey A, Lonsdale DM. Introns and their positions affect the translational activity of mRNA in plant cells. EMBO Rep 2001; 2: 394-8.

[47] Rose $\mathrm{AB}$. The effect of intron location on intron-mediated enhancement of gene expression in Arabidopsis. Plant J 2004; 40: 744-51.

[48] Toki S, Takamatsu S, Nojiri C, et al. Expression of a maize ubiquitin gene promoter-bar chimeric gene in transgenic rice plants. Plant Physiol 1992; 100: 1503-7.

[49] Shirasu K, Nielsen K, Piffanelli P, Oliver R, Schulze-Lefert P. Cell-autonomous complementation of mlo resistance using a biolistic transient expression system. Plant J 1999; 17: 293-9.

[50] Hayama R, Yokoi S, Tamaki S, Yano M, Shimamoto K. Adaptation of photoperiodic control pathways produces short-day flowering in rice. Nature 2003; 422: 719-22.

[51] Miki D, Shimamoto K. Simple RNAi vectors for stable and transient suppression of gene function in rice. Plant Cell Physiol 2004; 45: 490-5.

[52] Hirai S, Oka S, Adachi E, Kodama H. The effects of spacer sequences on silencing efficiency of plant RNAi vectors. Plant Cell Rep 2007; 26: 651-59.

[53] Nakayashiki H, Hanada S, Quoc NB, Kadotani N, Tosa Y, Mayama S. RNA silencing as a tool for exploring gene function in ascomycete fungi. Fungal Genet Biol 2005; 42: 275-83.

[54] McGinnis K, Murphy N, Carlson AR, et al. Assessing the efficiency of RNA interference for maize functional genomics. Plant Physiol 2007; 143: 1441-51.

[55] Xiong AS, Yao QH, Peng RH, Li X, Han PL, Fan HQ. Different effects on ACC oxidase gene silencing triggered by RNA interference in transgenic tomato. Plant Cell Rep 2005; 23: 639-46.

[56] Rutherford G, Tanurdzic M, Hasebe M, Banks JA. A systemic gene silencing method suitable for high throughput, reverse genetic analyses of gene function in fern gametophytes. BMC Plant Biol 2004; 16: 4-6.

[57] Sin SF, Yeung EC, Chye ML. Downregulation of Solanum americanum genes encoding proteinase inhibitor II causes defective seed development. Plant J 2006; 45: 58-70.

[58] Liu Q, Singh S, Green A. High-oleic and high-stearic cottonseed oils: nutritionally improved cooking oils developed using gene silencing. J Am Coll Nutr 2002; 21: 205-11.

[59] Rohr J, Sarkar N, Balenger S, Jeong BR, Cerutti H. Tandem inverted repeat system for selection of effective transgenic RNAi strains in Chlamydomonas. Plant J 2004; 40: 611-21.

[60] Li J, Brunner AM, Shevchenko O, et al. Efficient and stable transgene suppression via RNAi in field-grown poplars. Transgenic Res 2008; 17: 679-94.

[61] Miki D, Itoh R, Shimamoto K. RNA silencing of single and multiple members in a gene family of rice. Plant Physiol 2005; 138: 1903-13.

[62] Travella S, Klimm TE, Keller B. RNA interference-based gene silencing as an efficient tool for functional genomics in hexaploid bread wheat. Plant Physiol 2006; 142: 6-20.

[63] Outchkourov NS, Peters J, de Jong J, Rademakers W, Jongsma MA. The promoter-terminator of chrysanthemum rbcS1 directs very high expression levels in plants. Planta 2003; 216: 1003-12.

[64] Mlynarova L, Hricova A, Loonen A, Nap JP. The presence of a chromatin boundary appears to shield a transgene in tobacco from RNA silencing. Plant Cell 2003; 15: 2203-17.

[65] van der Geest AH, Welter ME, Woosley AT, et al. A short synthetic MAR positively affects transgene expression in rice and Arabidopsis. Plant Biotechnol J 2004; 2: 13-26. 
[66] Abranches R, Shultz RW, Thompson WF, Allen GC. Matrix attachment regions and regulated transcription increase and stabilize transgene expression. Plant Biotechnol J 2005; 3: 535-43.

[67] Halweg C, Thompson WF, Spiker S. The rb7 matrix attachment region increases the likelihood and magnitude of transgene expression in tobacco cells: a flow cytometric study. Plant Cell 2005; 17 : 418-29.

[68] Sotirova VN, Calciano MA, Krueger W, Lalande M. Inclusion of a matrix-attached region in a 7SK pol III vector increases the efficiency of shRNA-mediated gene silencing in embryonic carcinoma cells. Plasmid 2006; 5: 216-26.

[69] Nowak W, Gawłowska M, Jarmołowski A, Augustyniak J. Effect of nuclear matrix attachment regions on transgene expression in tobacco plants. Acta Biochim Pol 2001; 48: 637-46.

[70] Van Leeuwen W, Mlynarova L, Nap JP, van der Plas LH, van der Krol AR. The effect of MAR elements on variation in spatial and temporal regulation of transgene expression. Plant Mol Biol 2001; 47: 543-54.

[71] Brouwer C, Bruce W, Maddock S, Avramova Z, Bowen B. Suppression of transgene silencing by matrix attachment regions in maize: a dual role for the maize 5' ADH1 matrix attachment region. Plant Cell 2002; 14: 2251-64.

[72] Torney F, Partier A, Says-Lesage V, Nadaud I, Barret P, Beckert M. Heritable transgene expression pattern imposed onto maize ubiquitin promoter by maize $a d h-1$ matrix attachment regions: tissue and developmental specificity in maize transgenic plants. Plant Cell Rep 2004; 22: 931-8.

[73] Llave C, Kasschau KD, Rector MA, Carrington JC. Endogenous and silencing-associated small RNAs in plants. Plant Cell 2002; 14 : 1605-19.

[74] Molnár A, Csorba T, Lakatos L, Várallyay É, Lacomme C, Burgyán J. Plant virus-derived small interfering RNAs originate predominantly from highly structured single-stranded viral RNAs. J Virol 2005; 79: 7812-18.

[75] Mi S, Cai T, Hu Y, et al. Sorting of small RNAs into Arabidopsis Argonaute complexes is directed by the 5 ' terminal nucleotide. Cell 2008; 133: 116-27.

[76] Hellens RP, Allan AC, Friel EN, et al. Transient expression vectors for functional genomics, quantification of promoter activity and RNA silencing in plants. Plant Methods 2005; 18: 1-13.

[77] Collier R, Fuchs B, Walter N, Lutke WK, Taylor CG. Ex vitro composite plants: an inexpensive, rapid method for root biology. Plant J 2005; 43: 449-57.

[78] Helliwell C, Waterhouse P. Constructs and methods for highthroughput gene silencing in plants. Methods 2003; 30: 289-95.

[79] Homepage on the Internet. Available from: http://invitrogen.com/ content.cfm

[80] Earley KW, Haag JR, Pontes O, et al. Gateway-compatible vectors for plant functional genomics and proteomics. Plant J 2006; 45: 616-29.

[81] Karimi M, Depicker A, Hilson P. Recombinational cloning with plant gateway vectors. Plant Physiol 2007; 145: 1144-54.

[82] Helliwell CA, Wesley SV, Wielopolska AJ, Waterhouse PM. Highthroughput vectors for efficient gene silencing in plants. Funct Plant Biol 2002; 29: 1217-25.

[83] Helliwell CA, Waterhouse PM. Constructs and methods for hairpin RNA-mediated gene silencing in plants. Methods Enzymol 2005; 392: 24-35.

[84] Yelin MD, Chung SM, Frankman EL, Tzfira T. pSAT RNA interference vectors: a modular series for multiple gene down-regulation in plants. Plant Physiol 2007; 145: 1272-81.

[85] Devaiah BN, Nagarajan VK, Raghothama KG. Phosphate homeostasis and root development in Arabidopsis are synchronized by the zinc finger transcription factor ZAT6. Plant Physiol 2007; 145: 147-59.

[86] Binarova P, Cenklova V, Prochazkova J, et al. $\gamma$-Tubulin is essential for acentrosomal microtubule nucleation and coordination of late mitotic events in Arabidopsis. Plant Cell 2006; 18: 1199-212.

[87] Lo C, Wang N, Lam E. Inducible double-stranded RNA expression activates reversible transcript turnover and stable translational suppression of a target gene in transgenic tobacco. FEBS Lett 2005; 579: 1498-502.

[88] Guo HS, Fei JF, Chua NH. A chemical-regulated inducible RNAi system in plants. Plant J 2003; 34: 383-92.
[89] Wielopolska A, Townley H, Moore I, Waterhouse P, Helliwell C. A high-throughput inducible RNAi vector for plants. Plant Biotechnol J 2005; 3: 583-90.

[90] Homepage on the Internet. Available for: http://www.pi.csiro.au/ RNAi/vectors.htm

[91] Craft J, Samalova M, Baroux C, et al. New pOp/LhG4 vectors for stringent glucocorticoid-dependent transgene expression in Arabidopsis. Plant J 2005; 41: 899-918.

[92] Schwab R, Ossowski S, Riester M, Warthmann N, Weigel D. Highly specific gene silencing by artificial microRNAs in Arabidopsis. Plant Cell 2006; 18: 1121-33.

[93] Chapman E, Carrington JC. Specialization and evolution of endogenous small RNA pathways. Nat Rev Genet 2007; 8: 884-96.

[94] Millar AA, Waterhouse PM. Plant and animal microRNAs: similarities and differences. Funct Integr Genomics 2005; 5: 129-35.

[95] Niu QW, Lin SS, Reyes JL, et al. Expression of artificial microRNAs in transgenic Arabidopsis thaliana confers virus resistance. Nat Biotecnol 2006; 24: 1420-8.

[96] Homepage on the Internet. Available from: http://wmd2. weigelworld.org/cgi-bin/mirnatools.pl

[97] Qu J, Ye J, Fang1 R. Artificial microRNA-mediated virus resistance in plants. J Virol 2007; 81: 6690-9.

[98] Homepage on the Internet. Available from: http://www. openbiosystems.com/RNAi/ArabidopsisthalianaamiRNA/

[99] Bucher E, Lohuis D, van Poppel PM, Geerts-Dimitriadou C, Goldbach R, Prins M. Multiple virus resistance at a high frequency using a single transgene construct. J Gen Virol 2006; 87: 3697-701.

[100] Xu P, Zhang Y, Kang L, Roossinck MJ, Mysore KS. Computational estimation and experimental verification of off-target silencing during posttranscriptional gene silencing in plants. Plant Physiol 2006; 142: 429-40.

[101] Ifuku K, Yamamoto Y, Sato F. Specific RNA interference in psbP genes encoded by a multigene family in Nicotiana tabacum with a short 3'-untranslated sequence. Biosci Biotechnol Biochem 2003; 67: 107-13.

[102] Fukusaki E, Kawasaki K, Kajiyama S, et al. Flower color modulations of Torenia hybrida by down-regulation of chalcone synthase genes with RNA interference. J Biotechnol 2004; 111: 229-40.

[103] Pawloski LC, Deal RB, McKinney EC, Burgos-Rivera B, Meagher $\mathrm{RB}$. Inverted repeat PCR for the rapid assembly of constructs to induce RNA interference. Plant Cell Physiol 2005; 46: 1872-8.

[104] Zhong R, Richardson EA, Ye ZH. Two NAC domain transcription factors, SND1 and NST1, function redundantly in regulation of secondary wall synthesis in fibers of Arabidopsis. Planta 2007; 225: 1603-11.

[105] Melquist S, Bender J. Transcription from an upstream promoter controls methylation signaling from an inverted repeat of endogenous genes in Arabidopsis. Genes Dev 2003; 17: 2036-47.

[106] Cigan AM, Unger-Wallace E, Haug-Collet K. Transcriptional gene silencing as a tool for uncovering gene function in maize. Plant $\mathrm{J}$ 2005; 43: 929-40.

[107] Heilersig BH, Loonen AE, Janssen EM, Wolters AM, Visser RG. Efficiency of transcriptional gene silencing of GBSSI in potato depends on the promoter region that is used in an inverted repeat. Mol Genet Genomics 2006; 275: 437-49.

[108] Fischer U, Kuhlmann M, Pecinka A, Schmidt R, Mette MF. Local DNA features affect RNA-directed transcriptional gene silencing and DNA methylation. Plant J 2008; 53: 1-10.

[109] Haley B, Zamore PD. Kinetic analysis of the RNAi enzyme complex. Nat Struct Mol Biol 2004; 11: 599-606.

[110] Fedorov Y, Anderson EM, Birmingham A, et al. Off-targeting by siRNA can induce toxic phenotype. RNA 2006; 12: 1188-96.

[111] Birmingham A, Anderson EM, Reynolds A, et al. 3' UTR seed matches, but not overall identity, are associated with RNAi offtargets. Nat Methods 2006; 3: 199-204.

[112] Jackson AL, Bartz SR, Schelter J, et al. Expression profiling reveals off-target gene regulation by RNAi. Nat Biotechnol 2003; 21 : 635-7.

[113] Qiu S, Adema CM, Lane T. A computational study of off-target effects of RNA interference. Nucleic Acids Res 2005; 33: 1834-47.

[114] Jackson AL, Burchard J, Schelter J, et al. Widespread siRNA "offtarget" transcript silencing mediated by seed region sequence complementarity. RNA 2006; 12: 1179-87.

[115] Jackson AL, Burchard J, Leake D, et al. Position-specific chemical modification of siRNA increases specificity of gene silencing. RNA 2006; 12: 1197-205. 
[116] Dietrich CR, Han G, Chen M, Berg RH, Dunn TM, Cahoon EB. Loss-of-function mutations and inducible RNAi suppression of Arabidopsis $L C B 2$ genes reveal the critical role of sphingolipids in gametophytic and sporophytic cell viability. Plant J 2008; 54: 28498 .

[117] Thomas CL, Jones L, Baulcombe DC, Maule AJ. Size constraints for targeting post-transcriptional gene silencing and for RNAdirected methylation in Nicotiana benthamiana using a potato virus $\mathrm{X}$ vector. Plant J 2001; 25: 417-25.

[118] Kumar D, Gustafsson C, Klessig1 DF. Validation of RNAi silencing specificity using synthetic genes: salicylic acid-binding protein 2 is required for innate immunity in plants. Plant J 2006; 45: 863-8.
[119] Baulcombe DC. Amplified silencing. Science 2007; 315: 199-200.

[120] Peterson BO, Albrechtsen M. Evidence implying only unprimed RdRP activity during transitive gene silencing in plants. Plant Mol Biol 2005; 58: 575-83.

[121] Shimamura K, Oka S, Shimotori Y, Ohmori T, Kodama H. Generation of secondary small interfering RNA in cell-autonomous and non-cell autonomous RNA silencing in tobacco. Plant Mol Biol 2007; 63: 803-13.

[122] Akashi H, Miyagishi M, Kurata H, Nagata T, Taira K. A simple and rapid system for the quantitation of RNA interference in plant cultured cells. Nucleic Acids Res Suppl 2001; 1:235-6.

(C) Hirai and Kodama; Licensee Bentham Open.

This is an open access article distributed under the terms of the Creative Commons Attribution License (http://creativecommons.org/licenses/by/2.5/), which permits unrestrictive use, distribution, and reproduction in any medium, provided the original work is properly cited. 